\title{
NOTE ON PSEUDO-VALUATION DOMAINS WHICH ARE NOT VALUATION DOMAINS
}

\author{
Tariq Shah and Waheed Ahmad Khan \\ Received: 3 December 2013; Revised: 27 March 2014 \\ Communicated by Pınar Aydoğdu
}

\begin{abstract}
In this article, we discuss the $n$-root closedness, root closedness, seminormality, $S$-root closedness, $S$-closedness, $F$-closedess of PVDs. A valuation domain, being integrally closed, is obviously root closed. So our interest of study is for a class of non-valuation PVDs. Let $R \subset B$ be a domain extension such that $R$ is a PVD and the common ideal $P$ of $R$ and $B$ is a prime ideal in $R$. If $R$ is $n$-root closed (respectively root closed, seminormal, $S$-root closed, $S$-closed, $F$-closed) in $B$, then $R / P$ is PVD, which is $n$-root closed (respectively root closed, seminormal, $S$-root closed, $S$-closed, $F$-closed) in $B / P$. Further we study the relationship of atomic PVDs to atomic PVDs, SHFDs, LHFDs and BVDs. We also discuss a relative ascent and descent in general and particularly for the antimatter property of PVDs.
\end{abstract}

Mathematics Subject Classification 2010: 13A15, 13A18, 13B30, 13F30

Keywords: PVD, atomic domain, $F+M$ construction, root-closed, factor ring, condition $*$, antimatter domain

\section{Introduction}

Following [3], an integral domain $R$ with quotient field $K$ is said to be root closed if whenever $x^{n} \in R$ for some $x \in K$, and $n \in \mathbb{Z}^{+}$, then $x \in R$. We define $R$ to be $n$-root closed if whenever $x^{n} \in R$ for some $x \in K$, then $x \in R$. Any integral domain $R$ is trivially 1-root closed. An integral domain $R$ is root closed if and only if it is $n$-root closed for each $n \in \mathbb{Z}^{+}$. Further, $R$ is seminormal if whenever $x \in K$ with $x^{2}, x^{3} \in R$, then $x \in R$. More generally, if $R \subseteq B$ be a unitary commutative ring extension, then $R$ is $n$-root closed in $B$ if whenever $x \in B$ with $x^{n} \in R$, then $x \in R$. By [19], $R$ is $F$-closed in $B$ if whenever $x \in B$ with $x^{2}, x^{3} \in R$ and $n x \in R$ for some $n \in \mathbb{Z}^{+}$, then $x \in R$. Following [8, Page 2], $A$ is S-root closed in $B$ if whenever $b$ is in $B$ and $b^{n}$ is in $A$ for some $n$ in $S$, then $b$ is in $A$. The ring $A$ is called $S$-closed in $B$ if $b$ is in $B$ and $b^{n}$ is in $A$ for all $n$ in $S$, then $b$ is in $A$.

According to Cohn [12], an integral domain $R$ is an atomic domain if each nonzero non-unit of $R$ is a product of a finite number of irreducible elements (atoms) of $R$. 
Acccording to [21], an atomic domain $R$ is a half-factorial domain $(H F D)$ if for each nonzero nonunit element $x \in R$, if $x=x_{1} \ldots x_{m}=y_{1} \ldots y_{n}$ with each $x_{i}, y_{j}, 1 \leq i \leq m$, $1 \leq j \leq n$ is irreducible element in $R$, then $m=n$.

According to [15], a prime ideal $P$ of an integral domain $R$ with quotient field $K$, is known as strongly prime if $x, y \in K$ such that $x y \in P$, then either $x \in P$ or $y \in P$. An integral domain $R$ is said to be pseudo-valuation domain $(P V D)$ if each prime ideal of $R$ is strongly prime.

This study includes an investigation of "When is a non-valuation PVD n-root closed (resp., root closed)?" Note that a valuation domain is a PVD (cf. [15, Proposition 1.1]). But a valuation domain is integrally closed and hence root closed, so our interest is in a $P V D$ which is not a valuation domain. In the first part of this study we establish some conditions under which a $P V D$ (which is not a valuation domain) is $n$-root closed (respectively root closed). We apply these results to obtain seminormal, $S$-root closed and $S$-closed $P V D$ s. We also discuss the behavior of root closure of $R / P$, where $P$ is a prime ideal of $R$, whenever $R$ is root closed $P V D$. In [8], $S$-root closure in factor ring of a root closed ring is discussed (cf. [8, Theorem $1.8]$ ), we extend it for $n$-root closure and for root closure. We also generalize [8, Proposition 1.5] for $n$-root closure and for root closure for extension of $P V D$ s.

According to [4], an $H F D$ is strongly half-factorial domain ( $S H F D$ ) if each of its overrings is an $H F D$. An $H F D$ is locally half-factorial domain $(L H F D)$ if each of its localization is an $H F D$. Following [16], let $R$ be an $H F D$ with quotient field $K$. If $R \neq K$, we define the boundary map $\delta_{R}: K^{*} \rightarrow \mathbb{Z}$ by $\delta_{R}(\alpha)=t-s$, where $\alpha=\left(x_{1} \ldots x_{t}\right) /\left(y_{1} \ldots y_{s}\right) \in K$ and $x_{i}, y_{j}$ are irreducible elements in $R$.

By [16], an integral domain $R$ with quotient field $K$, is called boundary valuation domain $(B V D)$ if $R$ is an $H F D$, and for any $\alpha \in K$ with $\delta_{R}(\alpha) \neq 0$, either $\alpha \in R$ or $\alpha^{-1} \in R$, where $\delta_{R}$ is boundary map defined on $K$.

We also discuss the relationship of atomic $P V D$ s in which we relate atomic $P V D$ s to $S H F D$ s, $L H F D$ s and $B V D$ s. Further we discuss a relative ascent and descent of $P V D$ s.

Finally we emphasize upon the antimatter property (a domain $R$ is an antimatter domain if it has no atoms [13]) of pseudo-valuation domain while considering the condition $*$. Here we generalized a few of results of [13] relative to the condition *. 


\section{Root closure in PVDs}

We begin with the following.

Remark 2.1. Let $V$ be a valuation domain of the form $K+M$, where $K$ is any field and $M$ is the maximal ideal of $V$. If $F$ is a proper subfield of $K$ which is $n$-root closed in $K$, then by [15, Example 2.1], $R=F+M$ is a PVD which is not a valuation domain. Since $V$ is a valuation domain and $F$ is $n$-root closed in $K$, therefore $R$ is $n$-root closed (cf. [3, Lemma 2.1 (c)]).

Let $S$ be a multiplicative submonoid of $\mathbb{P}$, generated by some set of positive primes. Here, an increasing sequence of subfields of $\mathbb{R}$ can be defined by $K_{0}=\mathbb{Q}$ and $K_{n+1}=K_{n}\left(\left\{x \in \mathbb{R} \mid x^{p} \in K_{n}\right.\right.$ for some $\left.\left.p \in \mathbb{P}\right\}\right) . K_{S}=\cup K_{n}$ is a field (see [6, $\mathrm{p}-7])$.

Remark 2.2. The additive monoid monoid $S=\mathbb{Q}^{+} \cup\{0\}$ form algebra $\mathbb{R}[X ; S]$ over $\mathbb{R}$, which is Bezeout domain (cf. [20, Example 4.5]). Let $P=\{f \in \mathbb{R}[X ; S]: f$ has zero constant term $\}. P$ is a prime ideal and clearly the multiplicative system $T=\mathbb{R}[X ; S] \backslash P$ is the set of elements with nonzero constant terms. It is easy to see that $\mathbb{R}[X ; S]=\mathbb{R}+P$ and as it is Bezeout domain, so $(\mathbb{R}[X ; S])_{T}=\mathbb{R}+(P)_{T}=V$ is a valuation domain with maximal ideal $(P)_{T}$ (cf. [20, Example 4.5]). Then $R=K_{S}+(P)_{T}$, where $K_{S}=\cup K_{n}$. Thus $R=K_{S}+(P)_{T}$ is n-root closed, as $K_{S}$ is $n$-root closed in $\mathbb{R}$, by $[6$, Lemma 3.2]. So, $R$ is $n$-root closed PVD which is not a valuation domain.

According to [20], an integral domain $R$, with quotient field $K$, is said to be pre-Schreier domain if for all $x, y, z \in R \backslash\{0\}, x \mid y z$ implies $x=r s$, where $r, s \in R$ with $r \mid y$ and $s \mid z$. An integrally closed pre-Schreier domain is called a Schreier domain.

Remark 2.3. In Remark 2.2, the maximal ideal $(P)_{T}$ is idempotent, therefore $R=K_{S}+(P)_{T}$ is pre-Schreier domain (cf. [20, Theorem 4.4]). As $K_{S}$ is not algebraically closed in $\mathbb{R}$, therefore $R$ is not integrally closed. Hence $R=K_{S}+(P)_{T}$ is an example of a pre-Schreier PVD which is not a Schreier domain but is root closed.

The above observations yield the following:

Lemma 2.4. Let $V$ be a valuation domain of the form $K+M$, where $K$ is a field and $M$ be the maximal ideal of $V$ and $F$ be a proper subfield of $K$.

(1) If $F$ is root closed in $K$, then $R=F+M$ is root closed PVD. 
(2) If $F$ is seminormal in $K$, then $R=F+M$ is seminormal $P V D$.

(3) If $F$ is $m, n$-root closed in $K$, then $R=F+M$ is mn-root closed.

(4) If $F$ is $S$-root closed in $K$, then $R=F+M$ is $S$-root closed $P V D$.

(5) If $F$ is $S$-closed in $K$, then $R=F+M$ is $S$-closed PVD.

Proof. (1) As $F$ is root closed, so $F$ is $n$-root closed for all $n \in \mathbb{Z}^{+}$. By Remark $2.1, R$ is $n$-root closed for all $n$. Hence $R$ is root closed.

(2) As $F$ is seminormal in $K$, so $F$ is 2,3-root closed in $K$. By Remark 2.1, $R$ is 2,3-root closed. Hence $R$ is seminormal.

(3) If $F$ is $m, n$-root closed, $K$ is $m n$-root closed [3, Lemma 2.1 (a)]. So $R$ is $m n$-root closed by Remark 2.1.

(4) Suppose $F$ is $S$-root closed, by definition of $S$-root closed if whenever $k \in K$ and $k^{n} \in F$ for all $n$ in $S$, implies $k \in F$ and by Remark 2.1, $R$ is $n$-root closed for some $n \in S$. Hence $R$ is $S$-root closed.

(5) As $F$ is $S$-closed in $K$, by definition of $S$-root closed if whenever $k \in K$ and $k^{n} \in F$ for all $n$ in $S$, implies $k \in F$ and by Remark 2.1, $R$ is $n$-root closed for all $n \in S$. Hence $R$ is $S$-closed.

Remark 2.5. $\quad$ (i) By [3, Example 2.2], let $L$ be the algebraic closure of $\mathbb{Q}$ and let $F$ be the subfield of $L$ consisting of all elements $\alpha$ over $\mathbb{Q}$ such that the minimal polynomial for $\alpha$ over $\mathbb{Q}$ is solvable. Choose $\beta \in L$ but not in $F$, then let $K=F(\beta)$. Next, let $V=K+M=K[[X]]$, where $K$ is a field, $M=X V$, then $R=F+M$ is root closed. Obviously by [11, Theorem 2.1], $R$ is one-dimensional Noetherian local domain which is not integrally closed.

(ii) Let $V=\mathbb{C}+M=\mathbb{C}[[\mathbb{X}]]$, where $M=X V$, then $D=\mathbb{R}+M$ is seminormal (cf. [7, Lemma 2.1$]$ ). It is not $n$-root closed $P V D$ for any $n>1$, since $\mathbb{C}$ contains $n$th root of unity, not in $\mathbb{R}$.

In [8], $S$-root closure of commutative ring extensions $R \subseteq B \subseteq C$ has already been discussed (see [8, Proposition 1.5]). We are looking at it for $n$-root closure and root closure particularly for the extensions of $P V D \mathrm{~s}$.

Proposition 2.6. Let $R \subseteq B \subseteq C$ be extensions of PVDs such that $B$ is n-root closed in $C$, then $R$ is n-root closed in $B$ if and only if $R$ is n-root closed in $C$.

Proof. Let $R \subseteq B \subseteq C$ be extensions of $P V D$ s such that $B$ is $n$-root closed in $C$. Let $R$ be $n$-root closed in $B$. This implies that for $x \in B$, if $x^{n} \in R$, then $x \in R$. Since $B \subseteq C$, therefore $x \in C$. So $R$ is $n$-root closed in $C$. Conversely, let $R$ be 
$n$-root closed in $C$, this means for any $x \in C, x^{n} \in R$ implies $x \in R$. So $x^{n} \in R \subseteq B$ shows that $x^{n} \in B$, where $x \in C$. Since $B$ is $n$-root closed in $C$, therefore $x \in B$. Hence $R$ is $n$-root closed in $B$.

Corollary 2.7. Let $R \subseteq B \subseteq C$ be extensions of PVDs.

(1) Let $B$ be root closed in $C$, then $R$ is root closed in $B$ if and only if $R$ is root closed in $C$.

(2) Let $B$ be seminormal in $C$, then $R$ is seminormal in $B$ if and only if $R$ is seminormal in $C$.

(3) Let $B$ be F-closed in $C$, then $R$ is $F$-closed in $B$ if and only if $R$ is $F$-closed in $C$.

Proof. (1) Let $R$ be root closed in $B$, that is $R$ is $n$-root closed in $B$ for all $n \in \mathbb{Z}^{+}$. For any $x \in B$, if $x^{n} \in R$, then $x \in R$, for all $n \in \mathbb{Z}^{+}$. This means $R$ is $n$-root closed in $C$ for all $n \in \mathbb{Z}^{+}$by Proposition 2.6. Hence $R$ is root closed in $C$. Conversely, let $R$ be root closed in $C$. This implies $R$ is $n$-root closed for all $n$. That is if $x^{n} \in R$, where $x \in C$, then $x \in R$ for all $n$. This means $R$ is $n$-root closed in $B$ for all $n \in \mathbb{Z}^{+}$, by Proposition 2.6. Hence $R$ is root closed in $B$.

(2) Let $R$ be seminormal in $B$. That is $x^{2}, x^{3} \in R$ implies $x \in R$, where $x \in B$. So $R$ is seminormal in $C$ by Proposition 2.6. Conversely, let $R$ be seminormal in $C$, that is, if $x^{2}, x^{3} \in R$, where $x \in C$, then $x \in R$. So $R$ is (2,3)-root closed in $B$, by Proposition 2.6. Hence $R$ is seminormal in $B$.

(3) Let $R$ be $F$-closed in $B$. That is for any $x \in B$ whenever $n x, x^{2}, x^{3} \in A$, where $n \in \mathbb{Z}^{+}$, then $x \in R$. Since $B \subseteq C$, so $x \in C$. That is for any $x \in C$ whenever $n x, x^{2}, x^{3} \in R$, then $x \in R$. Hence $R$ is $F$-closed in $C$. Conversely, let $R$ be $F$-closed in $C$. This implies whenever $x^{2}, x^{3}, n x \in R$, then $x \in R$, where $x$ $\in C$ and . Since $x^{2}, x^{3}, n x \in R \subseteq B$, so $x^{2}, x^{3}, n x \in B$, where $x \in C$. Also $B$ is $F$-closed in $C$, which implies that $x \in B$. Hence $R$ is $F$-closed in $B$.

In [8], it is established that, for a commutative ring extension $R \subseteq B$, the factor ring $R / I$ is $S$-root closed, where $R$ is a root closed ring and $I$ is a common ideal of $R$ and $B$ (see [8, Theorem 1.8]). We focus on this situation for prime ideal $P$ of $R$ which is also an ideal in $B$, instead of $I$ and discussed the root closure of factor ring $R / P$, whenever $R$ is root closed $P V D$. Furthermore we also address the seminormality and $F$-closedness for factor ring $R / P$ of a $P V D R$.

In Theorem 2.8, we prove the result specially for pseudo-valuation domains. 
Theorem 2.8. Let $B$ be a domain extension of a $P V D R$ such that $P$ is a prime ideal of $R$ which is also an ideal in $B$. If $R$ is n-root closed in $B$, then $R / P$ is $P V D$, which is n-root closed in $B / P$.

Proof. By [10, Corollary 3], $R / P$ is $P V D$. Let $(x+P)^{n} \in R / P$, where $x+P \in$ $B / P$. This implies $x^{n}+P \in R / P$, where $x^{n} \in R$. Since $R$ is $n$-root closed in $B$, so $x^{n} \in R$ implies $x \in R$. That is $x+P \in R / P$. Hence $R / P$ is $n$-root closed in $B / P$.

Corollary 2.9. Let $B$ be a domain extension of a $P V D R$ such that $P$ is a prime ideal of $R$ which is also an ideal in $B$. Then

(1) $R$ is root closed in $B \Longrightarrow R / P$ is $P V D$, which is root closed in $B / P$.

(2) $R$ is seminormal in $B \Longrightarrow R / P$ is $P V D$, which is seminormal in $B / P$.

Proof. (1) $R$ is $n$-root closed in $B$ for all $n \in \mathbb{Z}^{+}$implies $R / P$ is $n$-root closed in $B / P$ for all $n$, by Theorem 2.8. This means $R / P$ is root closed in $B / P$.

(2) $R$ is seminormal in $B$ means $R$ is 2,3 -root closed in $B$. By Theorem 2.8, $R / P$ is 2,3 -root closed in $B / P$ implies $R / P$ is seminormal in $B / P$.

Theorem 2.10. Let $B$ be a domain extension of a $P V D R$ such that $P$ is a prime ideal of $R$ which is also an ideal in $B$. If $R$ is $S$-root closed in $B$, then $R / P$ is $P V D$, which is $S$-root closed in $B / P$.

Proof. By [10, Corollary 3] $R / P$ is a $P V D$. Let $(x+P)^{n} \in R / P$, where $x+P \in$ $B / P$ for some $n \in S$. This implies $x^{n}+P \in R / P$, that is $x^{n} \in R$ for some $n \in S$. Since $R$ is $S$-root closed, so $x \in R$ and $x+P \in R / P$. Hence $R / P$ is $S$-root closed in $B / P$.

Corollary 2.11. Let $B$ be a domain extension of a $P V D R$ such that $P$ is a prime ideal of $R$ which is also an ideal in $B$. If $R$ is $S$-closed in $B$, then the $P V D R / P$ is $S$-closed in $B / P$.

Proof. Let $R$ be $S$-root closed in $B$ for all $n \in S$. By Theorem 2.10, $R / P$ is $S$-root closed in $B / P$ for all $n \in S$. Hence $R / P$ is $S$-closed in $B / P$.

Theorem 2.12. Let $B$ be a domain extension of a $P V D R$ such that $P$ is a prime ideal of $R$ which is also an ideal in $B$. If $R$ is F-closed in $B$, then $R / P$ is $P V D$, which is F-closed in $B / P$.

Proof. Let $R \subseteq B$ be a ring extension, where $R$ is a $P V D$ which is $F$-closed in $B$. $R / P$ is a $P V D([10$, Corollary 3$]) . R$ is $F$-closed in $B$ implies if whenever $x \in B$ 
with $x^{2}, x^{3} \in R$ and $n x \in R$ for some positive integer $n$, then $x \in R$. Let $(x+P)^{2}$, $(x+P)^{3} \in R / P$. This means $x^{2}+P, x^{3}+P \in R / P$. Let $n(x+P) \in R / P$, then $n x+P \in R / P$, where $x+P \in B / P$ and so $n x \in R$. As $R$ is $F$-root closed, so $x \in R$, which implies that $x+P \in R / P$. Hence $R / P$ is $F$-closed in $B / P$.

\section{Atomic PVDs}

In this part we consider the case of atomic $P V D \mathrm{~s}$ and relate it with $S H F D \mathrm{~s}$, $L H F D \mathrm{~s}$ and $B V D \mathrm{~s}$.

Recall from [4] that, an $H F D$ is $S H F D$ if each of its overring is an $H F D$.

Proposition 3.1. A Noetherian PVD is an SHFD.

Proof. Each overring of $R$ is $P V D$ (cf. [15, Corollary 3.3]). Since $R$ is Noetherian, therefore its integral closure $R^{\prime}$ is Noetherian and $\operatorname{dim}\left(R^{\prime}\right)$ is 1 (cf. [15, Corollary 3.4]). Obviously $R^{\prime}$ is a Dedekind domain. By [14, Theorem 1], every overring of $R$ is atomic. That is each overring of $R$ is an atomic $P V D$. So each overring of $R$ is an HFD by [9, Theorem 5]. Hence $R$ is an SHFD.

Following [4], an $H F D$ is $L H F D$ if each of its localization is an $H F D$.

Remark 3.2. By definitions of $S H F D$ and $L H F D$ it is clear that an $S H F D$ is an LHFD. Therefore a Noetherian PVD is also an LHFD because it is an SHFD by Proposition 3.1.

Remark 3.3. (i) Let $(R, M)$ be a PVD which is not a valuation domain and $M^{2}=M$, then $R$ is a pre-Schreier [20, Theorem 4.4].

(ii) An atomic PVD may not be a finite factorization domain (FFD), (By [1], $R$ is a finite factorization domain (FFD) if each nonzero non-unit element of $R$ has a finite number of non-associate divisors and hence, only a finite number of factorizations upto order and associates), because $\mathbb{R}+X \mathbb{C}[[X]]$ is an atomic $P V D$ which is not an FFD.

Following [16], let $R$ be an $H F D$ with quotient field $K$. If $R \neq K$, we define the boundary map $\delta_{R}: K^{*} \rightarrow \mathbb{Z}$ by $\delta_{R}(\alpha)=t-s$, where $\alpha=\left(x_{1} \ldots x_{t}\right) /\left(y_{1} \ldots y_{s}\right) \in K$ and $x_{i}, y_{j}$ are irreducible elements in $R$.

Recall from [16] that, an integral domain $R$ with quotient field $K$, is called $B V D$ if $R$ is an HFD and for any $\alpha \in K$ with $\delta_{R}(\alpha) \neq 0$ either $\alpha \in R$ or $\alpha^{-1} \in R$, where $\delta_{R}$ is boundary map defined on $K$. 
Theorem 3.4. The following assertions are equivalent for an integral domain $R$.

(1) $R$ is an atomic $P V D$.

(2) $R$ is a $B V D$.

Proof. $(1) \Rightarrow(2)$. Let $R$ with quotient field $K$, be an atomic $P V D$. This implies $R$ is an HFD by [9, Theorem 5]. Let $\delta_{R}: K^{*} \longrightarrow \mathbb{Z}$ be a boundary map defined on $K^{*}=K \backslash\{0\}$ as $\delta_{R}(\alpha)=t-s$, where $x / y=\alpha=\left(x_{1} \ldots x_{t}\right) /\left(y_{1} \ldots y_{s}\right)$ and $x_{i}, s$, $y_{j, s}$ are irreducible elements in $R$. Assume $\delta_{R}(\alpha) \neq 0$. If $\delta_{R}(\alpha)<0$, then $t-s<0$, that is $t<s$, by [9, Theorem 6] $x \mid y$, thus $y / x \in R$ gives $\alpha^{-1} \in R$. If $\delta_{R}(\alpha)>0$, then $t-s>0$, that is $t>s$, by [9, Theorem 6] $y \mid x$, thus $x / y \in R$, which shows that $\alpha \in R$. Hence $R$ is a $B V D$.

$(2) \Rightarrow(1)$. Let $R$ be a $B V D$, so $\delta_{R}(x)<\delta_{R}(y)$ implies $x \mid y$ (cf. [16, Theorem 2.3]). Thus by $[9$, Theorem 6$] R$ is a $P V D$.

Corollary 3.5. If $R$ is a Noetherian PVD, then each overring of $R$ is a BVD.

Proof. Let $R$ be a Noetherian PVD. Each overring of $R$ is an HFD, by Proposition 3.1. Also $R$ is a $B V D$ by Theorem 3.4. Each overring of $R$ is $B V D$ (cf. [16, Theorem 3.3]).

\section{A relative ascent and descent}

We recall the following as in [17].

Condition $*$ : Let $R \subseteq B$ be a unitary (commutative) ring extension such that $U(B)$ represent the set of units of $B$. For each $b \in B$ there exist $u \in U(B)$ and $a \in R$ such that $b=u a$.

For a unitary (commutative) ring extension $R \subseteq B$, the conductor of $A$ in $B$ is the largest common ideal $R: B=\{a \in R: a B \subseteq R\}$ of $R$ and $B$.

The following are few examples of unitary (commutative) ring extensions which are satisfying Condition $*$.

Remark 4.1. [17, Example 1]

(i) If $B$ is a field, then the ring extension $R \subseteq B$ satisfies Condition *.

(ii) If $B$ is a fraction ring of $R$, then the ring extension $R \subseteq B$ satisfies Condition *. Hence the ring extension $R \subseteq B$ satisfies Condition * is the generalization of localization.

(iii) If the ring extensions $R \subseteq B$ and $B \subseteq C$ satisfy Condition $*$, then so does the ring extension $R \subseteq C$.

(iv) If the ring extension $R \subseteq B$ satisfies Condition *, then the extensions of rings $R+X B[X] \subseteq B[X]$ and $R+X B[[X]] \subseteq B[[X]]$ satisfy Condition $*$. 
There are number of examples of domain extensions $R \subseteq B$ which are satisfying Condition * but the conductor ideal $R: B$ is not a maximal ideal of $R$, as the following remark shows.

Remark 4.2. (i) Following [2, Example 5.3], let $V$ be a valuation domain such that its quotient field $K$ is the countable union of an increasing family $\left\{V_{i}\right\}_{i \in I}$ of valuation overrings of $V$. Let $L$ be a proper field extension of $K$ thus, $L^{*} / K^{*}$ is infinite as indicated in [2, Example 5.3].

(a) The domain extension $R=V_{i}+X L[[X]] \subseteq L[[X]]=B$ satisfies the Condition $*$ as the extension $V_{i} \subseteq L$ satisfies the Condition *. But $X L[[X]]$ is not a maximal ideal in $R$ and such that $U(R) \neq U(B)$.

(b) The domain extension $R=V_{i}+X L[[X]] \subseteq K+X L[[X]]=C$ satisfies the Condition * but $X L[[X]]$ is not a maximal ideal in $R$ and such that $U(R) \neq U(C)$.

(ii) The domain extension $R=\mathbb{Z}_{(2)}+X \mathbb{R}[[X]] \subseteq \mathbb{Q}+X \mathbb{R}[[X]]=B$ satisfies the Condition $*$, indeed; as $\mathbb{Z}_{(2)} \subseteq \mathbb{Q}$ satisfies Condition $*$, so if $f(X)=$ $q+X \sum_{i=0}^{\infty} r_{i} X^{i} \in B$, then $q=q^{\prime} q^{\prime \prime}$, where $q^{\prime} \in U(B)=\mathbb{Q} \backslash\{0\}, q^{\prime \prime} \in \mathbb{Z}_{(2)}$, hence $f(X)=q^{\prime}\left(q^{\prime \prime}+X \sum_{i=0}^{\infty}\left(q^{\prime}\right)^{-1} r_{i} X^{i}\right)$, where $q^{\prime \prime}+X \sum_{i=0}^{\infty}\left(q^{\prime}\right)^{-1} r_{i} X^{i} \in$ $R$. But the conductor ideal $R: B$ is not a maximal ideal in $R$.

(iii) The domain extension $R=\mathbb{Z}_{(2)}+X \mathbb{R}[[X]] \subseteq \mathbb{R}[[X]]=B$, satisfies Condition * but the conductor ideal $R: B$ is not a maximal ideal in $R$.

4.1. The case of PVDs. In the following we observe that the ascent of $P V D$ holds for a domain extension $R \subseteq B$ which satisfies Condition *.

Theorem 4.3. Let $R \subseteq B$ be a domain extension which satisfies Condition *. If $R$ is a $P V D$, then $B$ is a $P V D$.

Proof. As $R$ is a $P V D$, [9, Proposition 4] says: A ring $R$ is a PVR iff for all $a, b \in R$ either $a \mid b$ or every proper divisor of $b$ divides $a$. Since $R$ is a $P V D$, therefore either $a \mid a_{1}$ or any divisor $d$ of $a_{1}$ divides $a$. Let $a \mid a_{1}$ clearly $b_{1} \mid b_{2}$. Now suppose $b_{1}$ does not divide $b_{2}$ and let $d \mid b_{2}$ which implies $d \mid a_{1} c_{1}$. As $c_{1}$ is unit, so $d \mid a_{1}$. Since $R$ is a $P V D$, therefore $d \mid a$, which implies that $d \mid a c$. That is $d \mid b_{1}$. Hence $B$ is a $P V D$.

Corollary 4.4. Let $R \subseteq B$ be a domain extension which satisfies Condition $*$ and $M=R: B$ be a maximal ideal of $R$. If $R$ is an atomic $P V D$, then $B$ is an atomic $P V D$.

Proof. Let $R$ be an atomic $P V D$. By [17, Proposition 2.6 (a)], $B$ is atomic. By Theorem 4.3, $B$ is $P V D$. Hence $B$ is an atomic $P V D$. 
Remark 4.5. The domain extensions $\mathbb{R}+X \mathbb{C}[[X]] \subseteq \mathbb{C}[[X]]$ and $\mathbb{Q}+X \mathbb{R}[[X]] \subseteq$ $\mathbb{R}[[X]]$ are examples on the Corollary 4.4 .

The following is an alternate approach to obtain as of Corollary 4.4. Although Theorem 4.6 has been proved in [18, Theorem 3.7], but still we are presenting here when $R$ is a pseudo-valuation domain.

Theorem 4.6. Let $R \subseteq B$ be domain extension which satisfies Condition $*$ and $M=R: B$ be a maximal ideal in $R$ where $K_{R}$ and $K_{B}$ are quotient fields of $R$ and $B$ respectively. If $R$ is a pseudo-valuation domain which is also a BVD then $B$ is also a pseudo-valuation which is $B V D$.

Proof. Suppose $R \subseteq B$ such that $R$ is a pseudo-valuation domain and also a bounded valuation domain $(B V D)$. Following Theorem $4.3, B$ is a pseudo-valuation domain. As a $B V D$ is an $H F D$, so $R$ is an $H F D$ and by [17, Theorem 2.6(e)] $B$ is an HFD. For $\alpha \in K_{B}^{*}$, we have $\alpha=\frac{b}{d}$, where $b, d \in B \backslash\{0\}$. This implies $\alpha=\frac{b}{d}=\frac{a b^{\prime}}{c d^{\prime}}$, where $a, c \in R, b^{\prime}, d^{\prime} \in U(B)$. This means $\alpha=\frac{b}{d}=\frac{a_{1} \ldots a_{s} b^{\prime}}{c_{1} \ldots c_{t} d^{\prime}}$, where $a_{1}, \ldots, a_{s}, c_{1}, \ldots, c_{t} \in R$ are irreducibles and by [17, Theorem 2.5(d)] also irreducibles in $B$. Obviously $u=\frac{b^{\prime}}{d^{\prime}} \in U(B)$ and therefore $\alpha=\frac{b}{d}=\frac{a}{c} u=\frac{a_{1} \ldots a_{s}}{c_{1} \ldots c_{t}} u$. Obviously $\delta_{B}(\alpha) \neq 0$ implies $\delta_{R}\left(\frac{a}{c}\right) \neq 0$ and therefore either $\frac{a}{c} \in R$ or $\frac{c}{d} \in R$. This implies $\frac{a}{c} u \in B$ or $\frac{c}{a} u^{-1} \in B$. Hence $B$ is a $B V D$.

4.2. The case of antimatter domains. If $R \subseteq B$ such that $R$ and $B$ are distinct domains with equal spectra, that is $\operatorname{Spec}(R)=\operatorname{Spec}(B)$, then by [5, Proposition 3.3] $R$ is quasi-local with maximal ideal $M$ and $R U(R)=M=B \backslash U(B)$. While considering equal spectra context it is easy to deal with antimatter domains in the same fashion as we dealt with atomic domains, we will utilize here.

The following lemma extended [13, Lemma 3.1] if we add the Condition *.

Lemma 4.7. Let $R \subseteq B$ be the domain extension such that $\operatorname{Spec}(R)=\operatorname{Spec}(B)$, which satisfies Condition $*$. Let $t \in B$ such that $t=$ ur, where $u \in U(B), r \in R$, then $t$ is an atom in $B$ if and only if $r$ is an atom in $R$.

Proof. By Condition $*$ each $t \in B$ can be written as $t=u r$, where $u \in U(B)$, $r \in R$. Let us suppose that $r \in R$ is the only atom of $R$, then $r$ can only be written as $r=r .1$. Then clearly $R$ is a quasilocal domain with maximal ideal $M$ generated by $r$. Then by [5, Proposition 3.3] we have $M=R U U(R)=B \backslash U(B)$. But $t=u r$, where $u \in U(B)$ and hence $t \in M$, which generates the maximal ideal $M$. Thus $t$ is an atom in $B$ if $r$ is an atom in $R$. 
The following proposition provides the sufficient condition for [13, Proposition $3.2(\mathrm{~b})]$.

Proposition 4.8. Let $R \subseteq B$ be the domain extension such that $\operatorname{Spec}(R)=$ $\operatorname{Spec}(B)$, which satisfies Condition $*$, then $B$ is an antimatter domain if and only if $R$ is an antimatter domain.

Proof. Assume that $R$ is an antimatter domain, then by Condition $*$ for $r \in R$ there exists $t \in B$ such that $t=u r$, with $u \in U(B)$ and $r \in R$. This means $t$ and $r$ are assosiates in divisibility in $B$ and by Lemma $4.7, t$ is not an atom in $B$. Thus $B$ is an antimatter domain. The converse follows by [13, Proposition 3.2(b)].

Lemma 4.9. Let $R$ be an integral domain with quotient field $F$ satisfying Condition

*. If $R$ is an antimatter domain, then any overring of $R$ having same spectrum to $R$ is an antimatter domain.

Proof. Let $R$ be an antimatter domain and $T$ be its overring such that $u \in U(T) \subseteq$ $F$ and each $t \in T$ can be written as $t=u r$ by Condition $*$. Then by Proposition 4.8 , it is clear that if $R$ is an antimatter domain then $T$ is an antimatter domain.

The following lemma is the converse of [13, Cor 3.3(b)].

Lemma 4.10. Let $R$ be a PVD and the extension $R \subseteq V$ satisfies Condition *, where $V$ is the canonically associated valuation overring of $R$. If $R$ is an antimatter domain, then $V$ is an antimatter domain.

Proof. The result is obvious by the irreducibility of an elements in $R$ and its overring $V$, by adding Condition $*$ gives the result that $V$ is an antimatter domain if $R$ is an antimatter domain.

Example 4.11. The domain extension $R=V_{i}+X L[[X]] \subseteq L[[X]]=B$ satisfies the Condition $*$ as the extension $V_{i} \subseteq L$ satisfies the Condition *. But $X L[[X]]$ is not a maximal ideal in $R$ and such that $U(R) \neq U(B)$. Then clearly if $R$ is antimatter domain then so is $B$, as irreducibility in $R$ implies irreducibility in $B$.

Example 4.12. Let $V=F+M$ be a nontrivial valuation domain, where $F$ is a field and $M$ the maximal ideal of $V$. Let $D$ be a domain with quotient field $F$ satisfying Condition $*$, and put $R=D+M$. Indeed $R$ is an antimatter domain if and only if $D$ is antimatter domain by $[13$, Corollary 3.10$]$ with Condition *.

Acknowledgment. The authors would like to thank the referee for the valuable suggestions and comments. 


\section{References}

[1] D. D. Anderson, D. F. Anderson and M. Zafrullah, Factorization in integral domains, J. Pure Appl. Algebra, 69 (1990), 1-19.

[2] D. D. Anderson, D. F. Anderson and M. Zafrullah, Factorization in integral domains, J. Algebra, 152 (1992), 78-93.

[3] D. F. Anderson, Root closure in integral domains, J. Algebra, 79 (1982), 51-59.

[4] D. F. Anderson and S. T. Chapman, Overrings of half-factorial domains II, Comm. Algebra, 23(11) (1995), 3961-3976.

[5] D. F. Anderson and D. E. Dobbs, Pairs of rings with the same prime ideals, Canad. J. Math, 32 (1980), 362-384.

[6] D. F. Anderson and D. E. Dobbs, Root Closure in Integral Domains III, Canad. Math. Bull., 41(1) (1998), 3-9.

[7] D. F. Anderson, D. E. Dobbs and J. A. Huckaba, On seminormal overrings, Comm. Algebra, 10 (1982), 1421-1448.

[8] D. F. Anderson, D. E. Dobbs and M. Roitman, Root closure in commutative rings, Ann. Sci. Univ. Clermont-Ferrand II Math., 26 (1990), 1-11.

[9] A. Badawi, Remarks on pseudo-valuation rings, Comm. Algebra, 28(5) (2000), 2343-2358

[10] A. Badawi, D. F. Anderson and D. E. Dobbs, Pseudo-valuation Rings, Proc. Second International conference on comm. rings, Lecture notes in Pure and applied Maths., 185 (1996), 57-67.

[11] E. Basttida and R.Gilmer, Overrings and divisorial ideals of rings of the form $D+M$, Michigan Math. J., 20 (1973), 79-95.

[12] P. M. Cohn, Bezeout rings and their subrings, Proc. Camb. Phil. Soc., 64 (1968), 251-264.

[13] J. Coykendall, D. E. Dobbs and B. Mullins, On integral domains with no atoms, Comm. Algebra, 27 (1999), 5813-5831.

[14] T. Dumitrescu, T. Shah and M. Zafrullah, Domains whose overrings satisfy ACCP, Comm. Algebra, 28(9) (2000), 4403-4409.

[15] J. R. Hedstrom and E. G. Houston, Pseudo-valuation domains, Pacific J. Math., 75(1) (1978), 137-147.

[16] J. Maney, Boundary valuation domains, J. Algebra, 273 (2004), 373-383.

[17] N. Radu, S. O. Ibrahim Al-Salihi and T. Shah, Ascend and descend of factorization properties, Rev. Roumaine Math. Pures Appl., 45(4) (2000), 659-669.

[18] T. Shah, Relative ascent and descent in a domain extension, Int. Electron. J. Algebra, 7 (2010), 34-46. 
[19] J. J. Watkins, Root and integral closure for $R[[X]]$, J. Algebra, 75 (1982), 43-58.

[20] M. Zafrullah, On a property of pre-Schreier domains, Comm. Algebra, 15(9) (1987), 1895-1920.

[21] A. Zaks, Half-factorial domains, Bull. Amer. Math. Soc., 82(5) (1976), 721-723.

\section{Tariq Shah}

Department of Mathematics

Quaid-I-Azam University

Islamabad, Pakistan

e-mail: stshah@gmail.com

\section{Waheed Ahmad Khan}

Department of Mathematics and Statistics

Caledonian College of Engineering

P O Box 2322, Seeb 111, Sultanate of Oman

e-mail: sirwak2003@yahoo.com 